\title{
Compensation Temperature of the Mixed-Spin Ising Model on the Hexagonal Lattice
}

\author{
W. Figueiredo, M. Godoy, and V. S. Leite \\ Departamento de Física, Universidade Federal de Santa Catarina 88040-900, Florianópolis, SC, Brazil
}

Received on 3 November, 2003

\begin{abstract}
We studied a layered mixed-spin Ising model, with spins $\sigma=1 / 2$ and $S=1$, distributed on the sites of a hexagonal lattice. For this spin arrangement, any spin at one lattice site has two nearest-neigbor spins of the same type, and four of the other type. We assumed that the exchange interaction between spins $\sigma$ and $S$ is antiferromagnetic, with the value $J_{1} . J_{2}$ is the exchange interaction between two nearest neighbor $\sigma$ spins, and $J_{3}$ is the coupling between two nearest neighbor $S$ spins. We also considered a single-ion crystal-field contribution $D$ to the $S$ sites. We performed mean-field calculations and Monte Carlo simulations to determine the compensation point of the model. We have shown that a compensation point can be present for any positive value of $D$. We have also found a negative lower bound for $D$, below which a compensation point can not appear. For each value of $D$, we determined the range of values of the $J_{2}$ and $J_{3}$ couplings for which a compensation point is realizable.
\end{abstract}

In the recent years a lot of efforts has been dedicated to the study of the ferrimagnetic materials, specially due to their great potential for technological applications [1, 2]. The search for the new designed ferrimagnetic materials requires also a better understanding of their physical properties. Despite their simplicity, mixed-spin Ising systems have shown to be the simplest models for studying ferrimagnetism. These models have been studied by a variety of techniques, including effective-field theories [3, 4], renormalization-group calculations [5, 6, 7] and Monte Carlo simulations $[8,9]$.

In a ferrimagnetic material two inequivalent moments, interacting antiferromagnetically, can give rise to a zero spontaneous magnetization below its critical temperature [10]. This happens, due to the different dependences of the sublattice magnetizations on temperature. Then, a special point can appear at a temperature below the critical one, where the sublattice magnetizations cancel exactly each other. This point is the so called compensation point. The magnetic behaviour of a ferrimagnetic system near the compensation point is of fundamental importance in the area of the thermomagnetic recording devices [11].

The compensation temperature of a mixed-spin Ising model on the square and honeycomb lattices was already investigated. In these lattices, the nearest-neigbour interactions are always between spins $\sigma$ and $S$. The mean-field approximation predicts the existence of a compensation point, but only for a very narrow region of negative values of the crystal-field parameter. However, these results are in disagreement with Monte Carlo simulations. It is necessary to include in the simulations a next-nearest ferromagnetic $\sigma-\sigma$ interaction, in order to have a compensation point.

In this work we investigated a layered mixed-spin Ising model with spins $\sigma=1 / 2$ and $S=1$, defined on a hexagonal lattice. The lattice is formed by alternate layers of spins $S$ and $\sigma$. The hamiltonian model for this ferrimagnetic system is

$$
\begin{gathered}
\mathcal{H}=-J_{1} \sum_{\langle i, j\rangle} S_{i} \sigma_{j}-J_{2} \sum_{\langle i, j\rangle} \sigma_{i} \sigma_{j} \\
-J_{3} \sum_{\langle i, j\rangle} S_{i} S_{j}-D \sum_{i} S_{i}^{2},
\end{gathered}
$$

where $J_{1}, J_{2}$ and $J_{3}$ are the exchange couplings between nearest-neighbor pairs of spins $\sigma-S, \sigma-\sigma$ and $S-S$, respectively, and $D$ is the crystal-field contribution. In all the following analysis we will take $J_{1}<0$. We have also performed mean field calculations at the one site level. For the Monte Carlo simulations, the initial configurations were taken random, and we flipped the spins once a time, according to the heat-bath algorithm [12]. We considered hexagonal lattices of lattice sizes $L$, along with periodic boundary conditions. We used lattices of sizes $L=8,16,32$ and 64 , and in each Monte Carlo step (MCs) we performed $L^{2}$ trials of flipping the spins. In order to get reliable results, we performed around $4000 \mathrm{MCs}$ for the lattice with $L=64$, where the first $1000 \mathrm{MCs}$ were discarded for the thermalization process. We also considered averages over 100 different samples in our calculations. Although not shown in the next figures, the error bars are smaller than the symbol sizes. Then, let us present our results.

In Fig. 1, we show the behavior of the sublattice magnetizations $\left(m_{\sigma}, m_{S}\right)$, and of the total magnetization, $m_{t o t}=$ $\left(m_{\sigma}+m_{S}\right) / 2$, as a function of the temperature. For the set of values of the exchange interactions, $J_{1}=-1.0 \mathrm{~J}$, $J_{2}=1.0 \mathrm{~J}$ and $J_{3}=-1.05 \mathrm{~J}$, and for the crystal-field pa- 
rameter $D=-0.75 \mathrm{~J}$, we observe the existence of a compensation point. Fig. 1(a) shows the results of the mean-field calculations, and we found $T_{c o m p}=0.78 \mathrm{~J} / k_{B}$ for the temperature of the compensation point, and $T_{c}=1.37 \mathrm{~J} / k_{B}$, for the transition temperature. As can be seen, the compensation point appears due to the different dependences of the sublattice magnetizations on temperature. As the temperature increases, more and more spins of the sublattice $S$ flip from their ordered to the disordered state, because of the antiferromagnetic exchange coupling $J_{3}$. However, as the ferromagnetic exchange interaction $J_{2}$ is positive, the majority of the spins of the sublattice $\sigma$ remains in its ordered state. At $T=T_{\text {comp }}$, the two sublattice magnetizations cancel each other, giving rise to a zero total magnetization. For $T>T_{\text {comp }}$, the total absolute magnetization increases up to a maximum value, and then starts to decrease, going to zero at the critical temperature $T_{c}$. Fig. 1(b), Monte Carlo simulations, presents the same trends of Fig. 1(a), but the results we found for the compensation and critical temperatures are $T_{\text {comp }}=0.26 \mathrm{~J} / k_{B}$ and $T_{c}=0.62 \mathrm{~J} / k_{B}$, respectively.
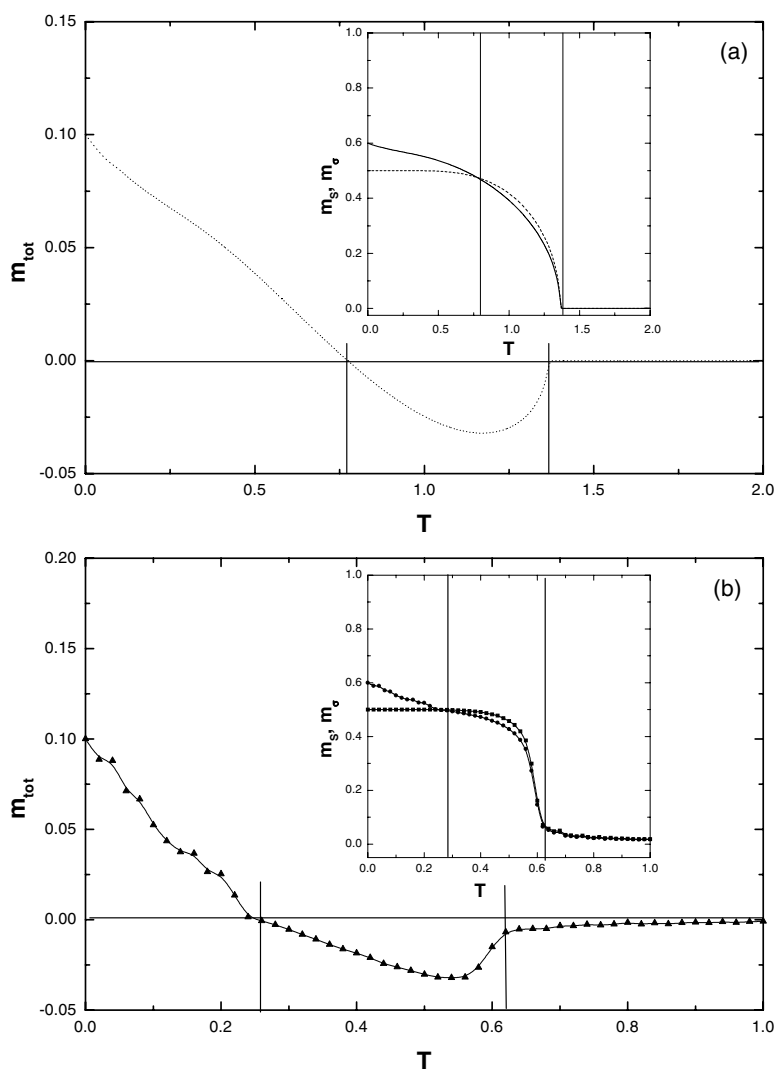

Figure 1. Sublattice and total magnetizations as a function of the temperature for $J_{1}=-1.0 \mathrm{~J}, J_{2}=1.0 \mathrm{~J}, J_{3}=-1.05 \mathrm{~J}$ and $D=-0.75 J$. (a) mean-field calculations and (b) Monte Carlo simulations. The insets show the sublattice magnetizations. $T_{\text {comp }}$ and $T_{c}$ are shown in the figures.

There is a minimum value of the ferromagnetic interaction parameter $J_{2}$ for the appearance of the compensation point, which depends on the value of the crystal-field $D$. We show in Fig. 2(a), the minimum value of $J_{2}$ as a function of $D$ in the mean-field approximation for $J_{1}=-1.0 \mathrm{~J}$ and
$J_{3}=-1.0 J$. The minimum value of $J_{2}$ for which a compensation point appears is an increasing function of $D$. As $D$ decreases, the magnetization of the sublattice $S$ decays faster, and the crossing point of the two sublattice magnetizations moves to lower temperatures. At these temperatures, the sublattice $\sigma$ is well ordered even for a small value of $J_{2}$. In Fig. 2(b) we present the dependence of $J_{2}$ on $D$, obtained through Monte Carlo simulations for $J_{1}=-1.0 \mathrm{~J}$ and $J_{3}=-0.98 J$. A different behavior appears in the range of values $-0.25 \mathrm{~J}<D<0.50 \mathrm{~J}$, where $J_{2}$ decreases with $D$. In this model, for $D<-3.00 J$, the system is represented by a set of uncoupled chains of $\sigma$ spins. We attribute this behavior to a dimensional crossover, which is not captured by the one site mean-field approximation.
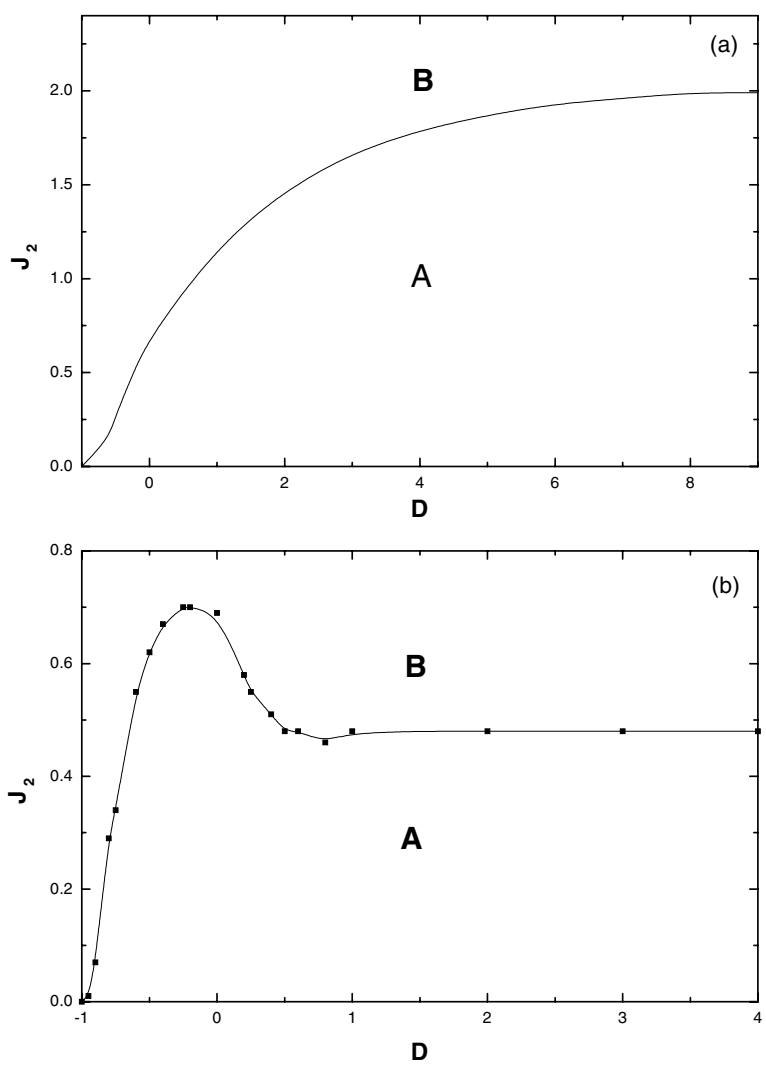

Figure 2. Minimum value of $J_{2}$ for the appearance of a compensation point as a function of the crystal field parameter $D$. (a) meanfield calculations for $J_{1}=-1.0 \mathrm{~J}$ and $J_{3}=-1.0$, and (b) Monte Carlo simulations for $J_{1}=-1.0 \mathrm{~J}$ and $J_{3}=-0.98$. Region $A$, $m_{s}>m_{\sigma}$ for $T<T_{c}$, and $B$ is the region where we have the compensation points.

Figure 3 shows the range of values of the antiferromagnetic interaction $J_{3}$ for which we have a compensation point, as a function of $D$, for $J_{1}=-1.0 \mathrm{~J}$ and $J_{2}=1.0 \mathrm{~J}$. Figs. 3(a) and 3(b) give the mean-field results and Monte Carlo simulations, respectively. In these figures, we note that there is a small region of values of $J_{3}$ for which we have a compensation point, region $B$ in the figures. In the region $A$ we always have $m_{S}>m_{\sigma}$ for any value of $T<T_{c}$. On the other hand, in the region $C, m_{\sigma}>m_{S}$ for any value of $T<T_{c}$. Monte Carlo simulations give a much more narrow 
range of values for $J_{3}$. Besides, the upper-bond $-J_{3}$ also exhibits a different behavior from the mean-field one in the range $-0.5<D<0$.
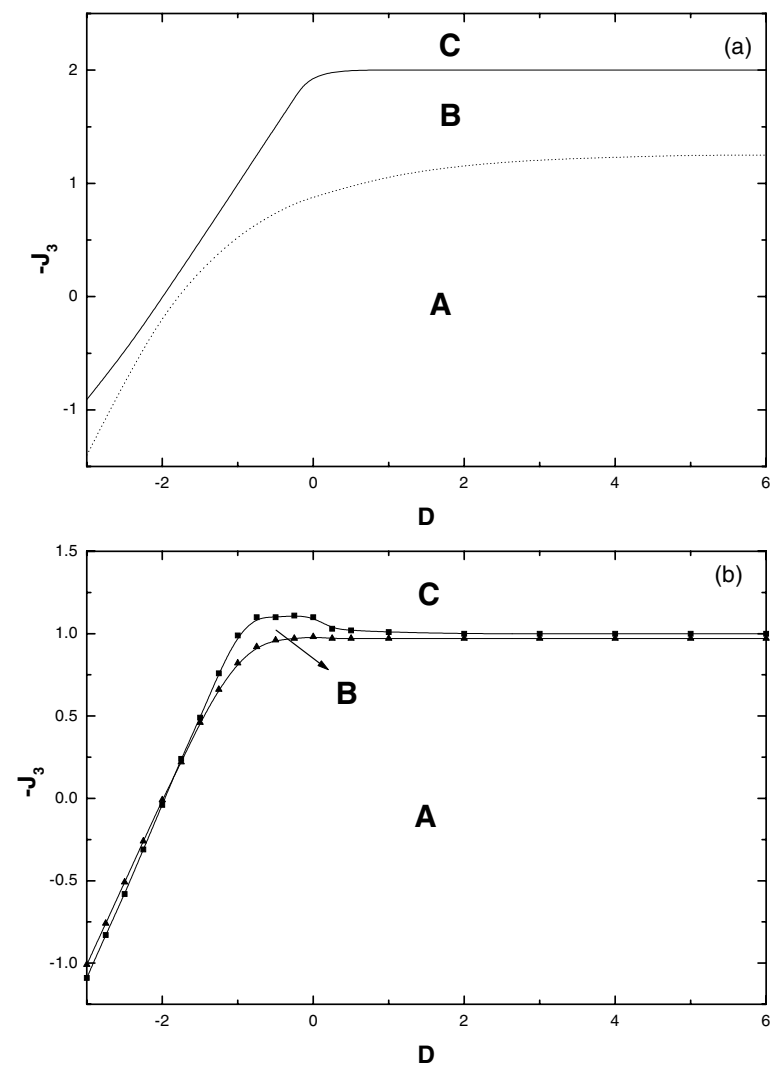

Figure 3. Range of values of $J_{3}$ giving rise the compensation points, as a function of the crystal field parameter $D$. (a) meanfield calculations, and (b) Monte Carlo simulations. $B$ is the only region where we can have the compensation points. For the regions $A$ and $C$, we always have $m_{s}>m_{\sigma}$, and $m_{\sigma}>m_{s}$, respectively.

In summary, we presented mean-field calculations and Monte Carlo simulations for a layered mixed-spin Ising model on a hexagonal lattice. We have shown that the model can exhibit a compensation point also for positive values of
$D$. We have also found a negative lower bound for $D$, below which there is no compensation point. For each value of $D$, we determined the range of values of $J_{2}$ and $J_{3}$ where the presence of a compensation point is possible.

\section{Acknowledgments}

This work was partially supported by the Brazilian agencies $\mathrm{CNPq}, \mathrm{CNPq}$ and FUNCITEC(SC).

\section{References}

[1] D. Gatteschi, O. Kahn, J. S. Miller and F. Palacio, Magnetic Molecular Materials (NATO ASI Series, Kluwer Academic, Dordrecht, 1991).

[2] H-P. D. Shieh and M. H. Kryder, Appl. Phys. Lett. 49, 473 (1986).

[3] T. Kaneyoshi, Solid State Communc. 70, 975 (1989); Physica A 205, 677 (1994).

[4] A. F. Siqueira and I. P. Fittipaldi, J. Magn. Magn. Mater. 54, 678 (1986).

[5] S. G. A. Quadros and S. R. Salinas, Physica A 206, 479 (1994).

[6] H. F. Verona de Resende, F. C. Sá Barreto and J. A. Plascak, Physica A 149A, 606 (1988).

[7] J. A. Plascak, W. Figueiredo and B. C. S. Grandi, Braz. J. Phys. 29, 579 (1999).

[8] G. M. Buendia and J. A. Liendo, J. Phys.: Condens. Matter. 9, 5439 (1997).

[9] G. M. Buendia, M. A. Novotny and J. Zhang. Springer Proceedings in Physics. Computer Simulation Studies in Condensed-Matter Physics VII. Eds. D. P. Landau, K. K. Mon and H. B. Schüttler. Springer-Verlag, 223-227 (1994).

[10] L. Néel, Ann. de Phys. 3, 137 (1948).

[11] M. Mansuripur, J. Appl. Phys. 61, 1580 (1987).

[12] D. P. Landau and K. Binder, A Guide to Monte Carlo Simulations in Statistical Physics (Cambridge University Press, United Kingdom, 2000). 